\title{
Permeability Prediction of Tight Sandstone Reservoirs Using Improved BP Neural Network
}

\author{
Peng Zhu ${ }^{1, *}$, Chengyan Lin ${ }^{1}$, Peng $\mathrm{Wu}^{2}$, Ruifeng Fan ${ }^{1}$, Hualian $\mathrm{Zhang}^{3}$ and Wei $\mathrm{Pu}^{4}$ \\ ${ }^{1}$ School of Geosciences in China University of Petroleum, Qingdao 266580, China; ${ }^{2}$ Binnan Oil Factory of Sinopec \\ Shengli Petrochemical Company, 256600, China; ${ }^{3}$ Chongqing Institute of Geology \& Mineral Resources, Chongqing, \\ 400042, China; ${ }^{4}$ Shixi Operation District, Xinjiang Oilfield Company, Petro China, Karamay, Xinjiang 834000, China
}

\begin{abstract}
By analyzing the permeability controlling factors of tight sandstone reservoir in Wuhaozhuang Oil Field, the permeability is considered to be mainly controlled by porosity, clay content, irreducible water saturation and diagenetic coefficient. Because the conventional BP algorithm has its drawbacks such as slow convergence speed and easy falling into the local minimum value, an improved three-layer feed-forward BP neural network model is built by MATLAB neural network toolbox to predict permeability according to the four permeability controlling factors, while studying samples of model are selected based on the representative core analysis data. The simulation based on improved neural network model shows that the improved model has a faster convergence speed and better accuracy. The consistency between model prediction value and lab test value is good and the mean squared error is less. Therefore, the new model can meet the needs of the development geology research of oil field better in the future.
\end{abstract}

Keywords: BP neural network, improved BP algorithm, network simulation training, permeability prediction.

\section{INTRODUCTION}

Tight sandstone reservoirs usually undergo complicated diagenesis and structural reconstruction throughout the long geological history. It is often characterized by poor physical properties, strong diagenetic intensity, complex pore structure and strong heterogeneity. It is hard to conduct identification, prediction and quantitative calculation of tight sandstone reservoir's petrophysical parameters [1-3]. The main role of logging interpretation is to accurately obtain petrophysical parameters based on the core and logging information and provide reliable basic data for subsequent reservoir evaluation and numerical simulation. It is necessary but challenging to determine petrophysical parameters of tight sandstone reservoirs more precisely based on the available logging data. Permeability is a key parameter in tight sandstone reservoir's evaluation, whose accuracy will directly affect the productivity determination and reservoir's heterogeneity research [4-8]. Based on the permeability controlling factors analysis, this paper took the tight sandstone reservoir from Wuhaozhuang Oil Field as an example and established permeability prediction model of tight sandstone reservoirs by using the modified BP neural network algorithm.

\section{THE ANALYSIS OF CONTROLLING FACTORS OF PERMEABILITY OF TIGHT SANDSTONE RES- ERVOIR}

For any kind of medium-high permeable sandstone reservoir, the distribution of sedimentary microfacies is vital on

*Address correspondence to this author at the School of Geosciences in China University of Petroleum, Qingdao 266580, China;

Tel: +8618765925521; E-mail: $65814011 @ q q . c o m$ controlling the reservoir's physical properties. As for tight sandstone reservoir, however, the diagenesis is always one of the most important controlling factors on its tightening [912]. Therefore, we should not simply establish uniform, hierarchical or regional models for permeability interpretation of tight sandstone reservoirs, the impact of diagenesis needs to be considered additionally. The key question is how to quantify the impact of diagenesis on permeability. The diagenesis coefficient is the parameter that reflects the degree of impact on original porosity of all types of diagenesis [13]. It fully reflects how the reservoir pore space is affected after series of diagenetic evolution. Generally the coefficient is between $0 \sim 1$, the higher the value is, the stronger the impact will be on the diagenesis which improves the overall reservoir's petrophysical properties. The weaker the impact is on the diagenesis which deteriorates the overall reservoir's petrophysical properties, the higher the porosity and permeability will be; the smaller the value is, the stronger the impact will be on the diagenesis which deteriorates the overall reservoir's petrophysical properties and the lower the porosity and permeability. Therefore, the change of petrophysical properties caused by diagenesis can quantitatively characterized by diagenetic coefficient.

Based on the petrophysical experiment and previous research achievements, the paper has applied porosity, clay content, irreducible water saturation and diagenetic coefficient to predict the permeability. Through polyfactorial analysis between four parameters and permeability, Table $\mathbf{1}$ shows that the correlation coefficients between five parameters are all higher than 0.5 and the diagenetic coefficient is 0.629, which further highlights its importance on controlling reservoir permeability. 
Table 1. Polyfactorial analysis on permeability.

\begin{tabular}{|c|c|c|c|c|c|}
\hline Parameter & Porosity & Clay Content & Irreducible Water Saturation & Diagenetic Coefficient & Permeability \\
\hline \hline Porosity & 1 & & & \\
\hline Clay Content & -0.470 & 1 & & \\
\hline Irreducible Water Saturation & -0.509 & 0.577 & -0.454 & 1 \\
\hline Diagenetic Coefficient & 0.621 & -0.681 & -0.558 & 0.629 \\
\hline Permeability & 0.753 & -0.551 & & 1 \\
\hline
\end{tabular}

Table 2. Data of training samples.

\begin{tabular}{|c|c|c|c|c|c|}
\hline Number of Sample & $\begin{array}{c}\text { Porosity } \\
(\%)\end{array}$ & $\begin{array}{c}\text { Clay Content } \\
(\%)\end{array}$ & $\begin{array}{c}\text { Irreducible Water } \\
\text { Saturation }(\%)\end{array}$ & $\begin{array}{l}\text { Diagenetic } \\
\text { Coefficient }\end{array}$ & $\begin{array}{c}\text { Permeability } \\
(\mathrm{mD})\end{array}$ \\
\hline 1 & 7.6 & 3.6 & 12.1 & 0.082 & 3.7 \\
\hline 2 & 15.1 & 6.5 & 9.6 & 0.067 & 4.1 \\
\hline 3 & 14.6 & 7.6 & 4.2 & 0.075 & 0.8 \\
\hline 6 & 17.5 & 18.9 & 21.8 & 0.038 & 3.3 \\
\hline 7 & 16.9 & 6.5 & 18.5 & 0.064 & 3.6 \\
\hline 8 & 9.3 & 5.4 & 9.2 & 0.061 & 2.6 \\
\hline 12 & 9.7 & 17.9 & 20.1 & 0.014 & 0.4 \\
\hline 13 & 15.7 & 19.2 & 22.6 & 0.008 & 1.0 \\
\hline 14 & 13.1 & 17.8 & 19.6 & 0.017 & 0.6 \\
\hline 15 & 13.4 & 16.5 & 17.6 & 0.019 & 0.6 \\
\hline 16 & 8.1 & 23.1 & 20.3 & 0.009 & 0.3 \\
\hline 17 & 7.7 & 24.9 & 24.3 & 0.01 & 0.4 \\
\hline 18 & 10.1 & 28.2 & 12.8 & 0.017 & 0.4 \\
\hline
\end{tabular}

\section{BP NEURAL NETWORK ALGORITHM AND ITS IMPROVEMENT}

BP algorithm, as a type of mathematical model of neural network, is suggested for solving weight optimization of multi-layer feed-forward neural network model. It is also one of the most widely used neural network algorithms and the most practical one in automatic control. However, it has defects such as easy falling into local minimum value and slow convergence rate. In order to adjust these two defects occur- ring in iterative process, Additional Momentum Method and Self-adaptive Learning Rate Method are adopted [14-18].

Based on back propagation method, using Additional Momentum Method, the change of every weight function will be added with a value that is proportional to the last weight function's change and creates new weight function's change. The detailed workflow is to overlay the current weight adjustment value with a portion of previous weight adjustment value by the error calculation, and takes it as the actual weight adjustment value, which is: 


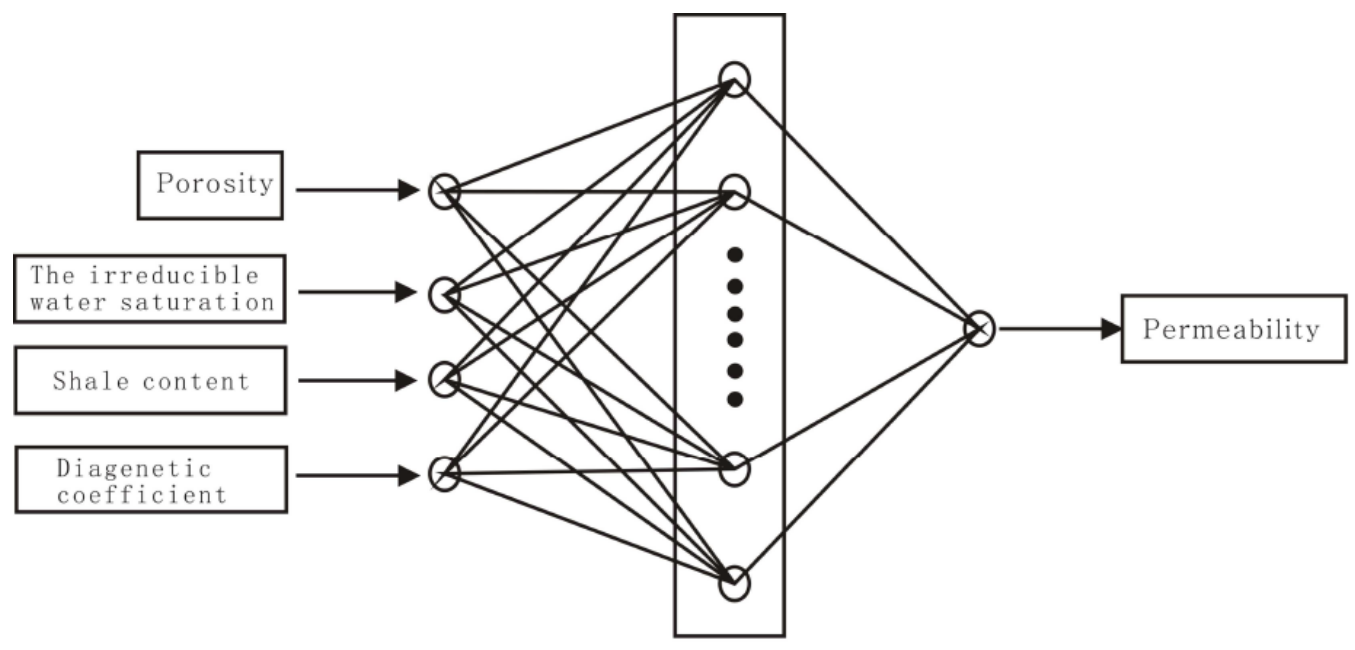

Input layer

Hidden layer

Ouput layer

Fig. (1). BP neural network model structure.
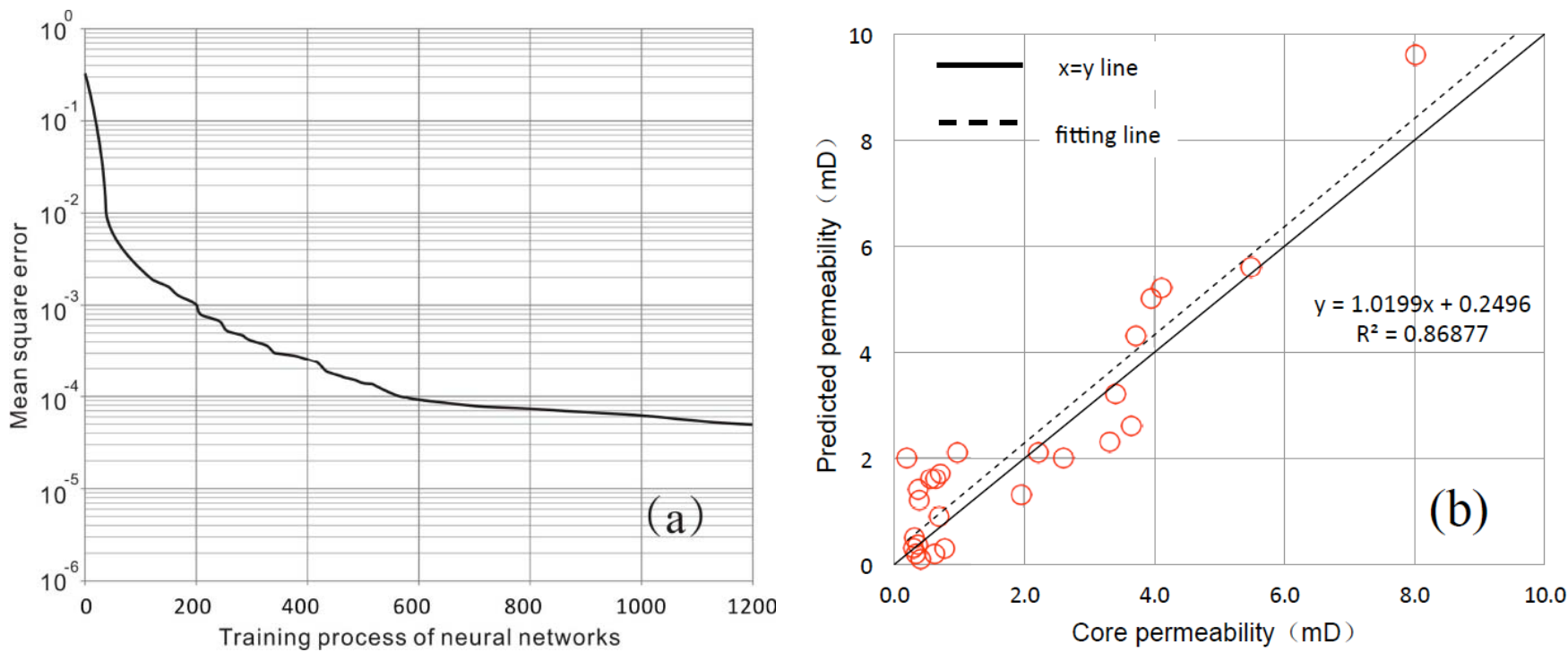

Fig. (2). The training process of conventional neural network model and the correlation between core permeability and predicted permeability.

$$
\Delta \omega_{i j}(n)=\alpha \Delta \omega_{i j}(n-1)+(1+\alpha) \eta \nabla f\left(\omega_{i j}(n-1)\right)
$$

Where, $\eta$ is the learning efficiency, whose range is between $0.001 \sim 10 ; n$ is the training times; $\alpha$ is the momentum factor, generally $0<\alpha<0.9 ; \nabla f\left(\omega_{i j}(n-1)\right)$ is the gradient of the error function.

The Additional Momentum Method is adopted to make the adjustment of the weight function change towards the direction of the average of error surface bottom. When the network weight functions enter the flat area, the occurrence of $\Delta \omega=0$ can be avoided and prevent the network from falling into the local minimum value of error surface.

The principle of Self-adaptive Learning Rate Method is that study rate will adjust and adapt on its own according to the change of error. By applying the Equation (2) during the training process, stability and convergence speed can be improved.

$$
\eta(n)=\left\{\begin{array}{cc}
1.05 \eta(n-1) & (E(n)<E(n-1)) \\
0.7 \eta(n-1) & (E(n)<1.04 E(n-1)) \\
\eta(n-1) & \text { else }
\end{array}\right.
$$

\section{THE ESTABLISHMENT OF PERMEABILITY PREDICTION MODEL AND ITS PRECISION ANALYSIS}

\subsection{Establishing Model}

The permeability prediction model is established by use of MATLAB neural network toolbox with a three-layered BP neural network structure. Training samples of tight sandstone are from one coring well of Wuhaozhuang Oil Field (Table 2). Porosity, clay content, irreducible water saturation and diagenetic coefficient are selected as the neural nodes of 

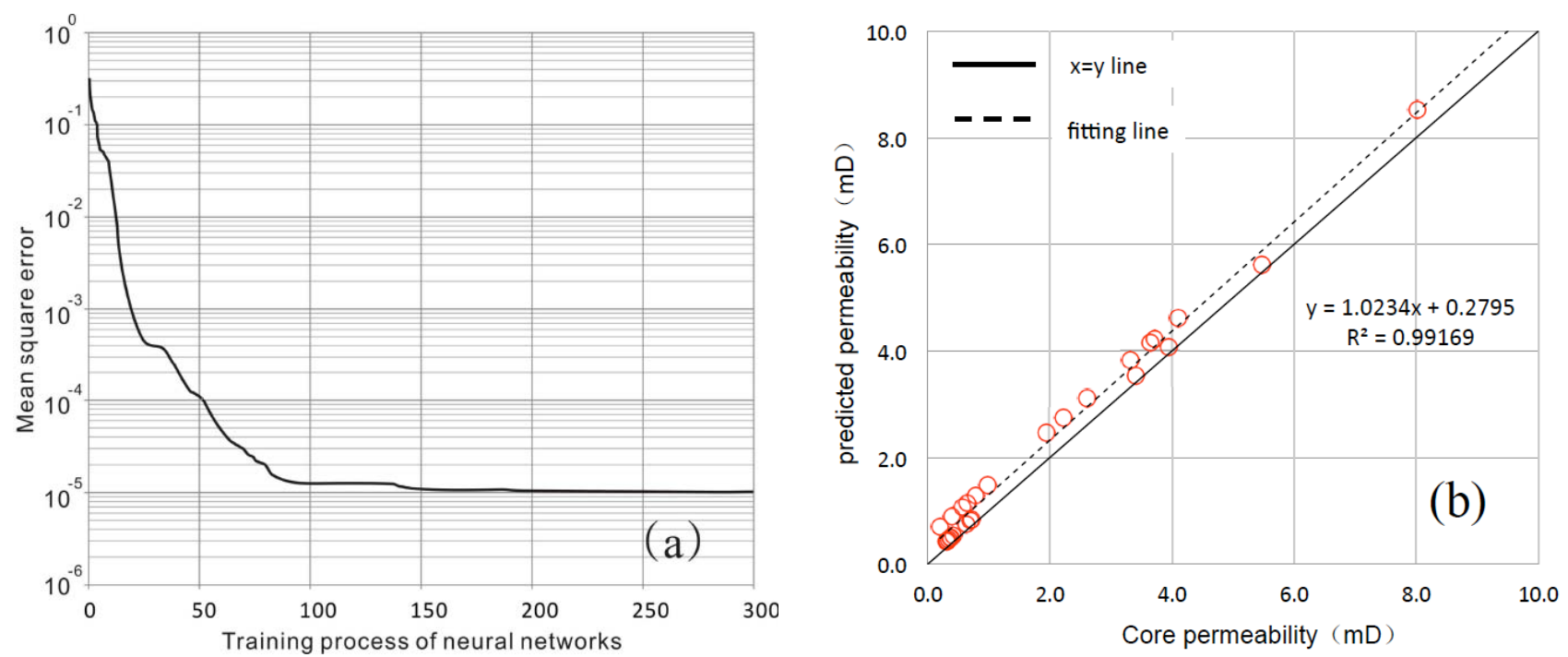

Fig. (3). The training process of improved neural network model and the correlation between core permeability and predicted permeability.

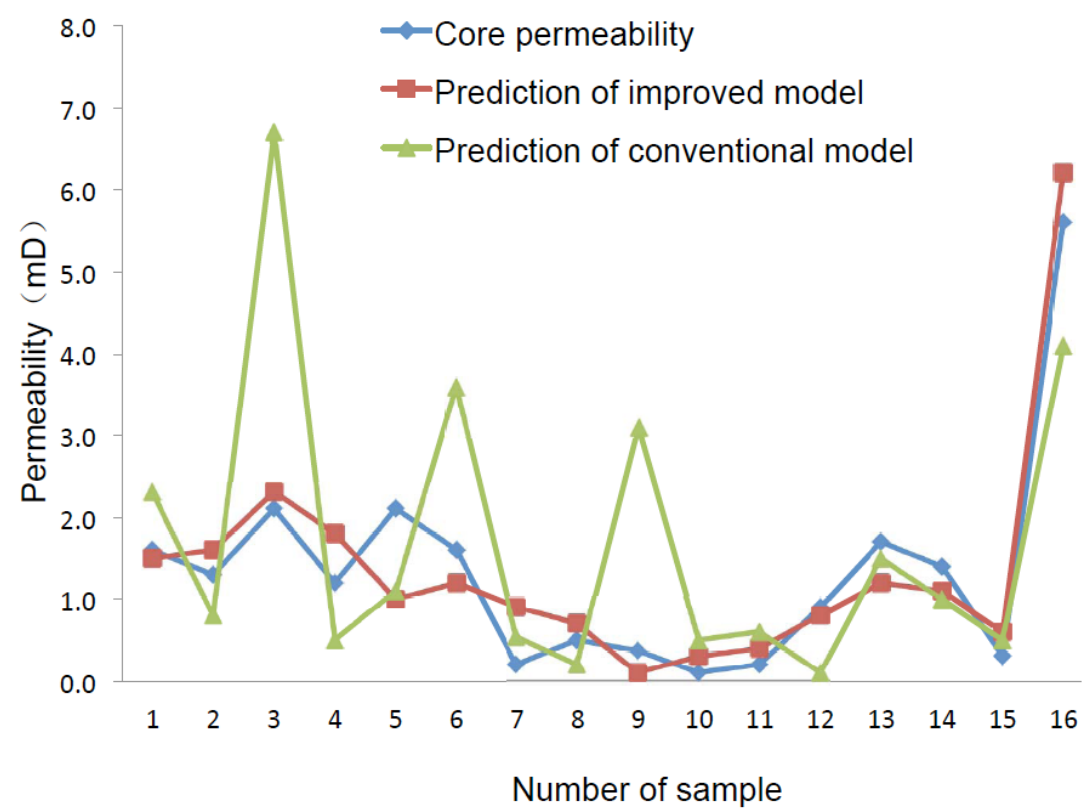

Fig. (4). Comparison between core permeability, the predicted permeability of improved model and the predicted permeability of conventional model.

input layer. The node value of output layer is set as 1 , representing permeability. And the number of hidden layer nodes is set as 20. The permeability prediction neural network structure is shown in Fig. (1). The minimum mean squared error, learning efficiency, momentum coefficient and maximum number of training are set as $0.00001,0.01,0.95$ and 15000. The order of samples has no impact on the results as the model applies group training. The training ends when the training number reaches to the maximum setting number or mean squared error threshold value. Take the mean squared error to test the generalization ability of the network, and obtain higher emulation neural network model via repeated training network. The simulation results are shown in the Fig. (2a) and Fig. (3a). It can be seen that after the original and improved model undergoing 600 and 80 time's network training, respectively, network errors (mean squared error) tend to be stable. The improved model shows sharp downward trend and its convergence rate is faster. As to the prediction accuracy, it can be seen from Fig. (2b) and Fig. (3b), by comparison, the predicted permeability of proved model is more fitting with core permeability.

\subsection{Model Accuracy Analysis}

According to the established high-emulation BP neural network model, the permeability of other 16 cores from the same reservoir has been predicted Fig. (4). By comparision with core permeability, the mean squared error of the improved model is 1.829 , while the mean squared error of the conventional model is 6.2. The results show that the accuracy is improved dramatically. 


\section{CONCLUSION}

The analysis of controlling factors of tight sandstone reservoir's petrophysical properties is the basis of establishing the prediction model. By selecting appropriate controlling factors, the accuracy of permeability prediction model is enhanced. The polyfactorial analysis is used to confirm that a strong non-linear relationship exists amongst porosity, clay content, irreducible water saturation, diagenetic coefficient and permeability, while the BP neural network has the ability to implement the complex non-linear mapping, which maks it the best option to solve the complex internal mechanism issues as these.

Applying Additional Momentum Method and Selfadaptive Learning Rate Method to modify BP algorithm can reduce the oscillation trend, increase its stability, improve the network convergence and enhance the prediction accuracy.

The prediction results show that the modified BP neural network model has less mean squared error than conventional model. In conclusion, the BP neural network with modified algorithm is more feasible on the prediction of tight sandstone permeability.

\section{CONFLICT OF INTEREST}

The authors confirm that this article content has no conflict of interest.

\section{ACKNOWLEDGEMENTS}

This work is sponsored by the Chinese National Science and Technology Major Project (No.2011ZX05009-003).

\section{REFERENCES}

[1] Y.F. Zeng, and W.J. Xia, Sedimentary Petrography. Geological Publishing House: Beijing 1985.

[2] B.J. Liu, and J.Q. Zhang, Sedimentary Diagenesis. Science Press: Beijing 1992.

[3] L.Y. Liu, and Z.L. Wang, "The diagenesis and lapidofacies of Yanchang Formation Chang 3 reserviors in Baibao Area", Journal of Northwest University: Natural Science Edition, vol. 38, no. 1, pp. 99-102, 2008.

[4] L. Shi, J.C. Kuang, J.Y. Zeng, X. Liu, X. Luo, and Z.H. Cai, "Single well productivity evaluation and prediction in tight clastic reservoir rock of Shaximiao formation in the Xinchang gas field, West
Sichuan”, Petroleum Geology \& Experiment, vol. 38, no. 1, pp. 99102, 2008.

[5] Z. Shi, Y.J. Shi, H.T. Zhang, T.D. Liu, and X.M. Yang, "Productivity prediction of tight sand reservoir with low permeability in Sulige Gas Field", Well Logging Technology, vol. 36, no. 6, pp. 641646, 2012.

[6] X.Q. Qiu, Z.P. Li, Y.S. Liu, and F.P. Lai, "Analysis of productivity equation and influence factors of horizontal wells in tight sand gas reserviors", Journal of Northwest University [Natural Science Edition], vol. 35, no. 2, pp. 142-145, 2013.

[7] Y. Hu, X.J. Li, Y.J. Wan, J.L. Lu, H.Y. Zhu, Y.F. Zhang, Q.Y Zhu, M. Yang, and L.W. Zhu, "Physical simulation on gas percolation in tight sandstone", Petroleum Exploration and Development, vol. 40, no. 5, pp. 580-584, 2013.

[8] J.C. Tian, W.W. Liu, F. Wang, R. Chen, and X.B. Lin, "Heterogeneity of the paleozoic tight sandstone reservoirs in Gaoqiao area of Ordo basin”, Oil \& Gas Geology, vol. 35, no. 2, pp. 183-189, 2014.

[9] Z.X. Lv, and S.B. Liu, "Ultra-tight sandstone diagenesis and mechanism for the formation of relatively high-quality reservoir of Xujiahe Group in western Sichuan”, Acta Petrologica Sinica, vol 25, no. 10, pp. 2373-2383, 2009.

[10] Y. Wang, Z.J. Shi, Y.W. Liu, and J.H. Wang, "Diagenesis of tight sandstone in the Silurian Xiaoheba formation of the Shizhu Synclinoyium, western Hubei-eastern Chongqing area", Oil \& Gas Geology, vol. 32, no. 1, pp. 75-82, 2011.

[11] R.C. Yang, X.P. Wang, A.P. Fan, Z.Z. Han, J. Zhao, and Y.L. Wang, "Diagenesis of sandstone and genesis of compact reservoirs in the east II part of Sulige gas field, Ordos basin", Acta Sedimentologica Sinica, vol. 30, no. 1, pp. 111-119, 2012.

[12] W. Zhang, F. Xu, G.S. Xu, W.Z. Gao, and J.C Tang, "Diagenesis and pore evolution of Huaguang formation tight sandstone reservoirs in a structure of Xihu depression in East China Sea basin", Journal of Chengdu University of Technology (Science \& Technology Edition), vol. 39, no. 2, pp. 122-129, 2012.

[13] Y. Cao, and G.X. Lv, "The effect of integrative modulus of diagenesis on reservoir distribution prediction", Xinjiang Oil \& Gas (Edition of Natural Science), vol. 3, no. 4, pp. 12-16, 2007.

[14] H.M. Goda, H.R. Maier, and P. Behrenbruch, "The Development of an Optimal Artificial Neural Network Model for Estimating Initial Water Saturation-Australian Reservoir", SPE93307, pp. 312-314, 2005.

[15] H. Li, and J.F. Yang, "Model reference adaptive control based on the improved BP neural network", Journal of Lanzhou Jiaotong University, vol. 30, no. 1, pp. 37-41, 2011.

[16] J. Yang, C.B. Yang, Y. Zhang, L.G. Cui, and L.F. Wang, "Permeability prediction method based on improved BP neural network", Lithologic Reservoirs, vol. 23, no. 1, pp. 98-102, 2011.

[17] Y.Q. Zhang, X. Liu, and X.Y. Sun, "An improved algorithm of BP neural network and its application", Computer Technology and Development, vol. 22, no. 8, pp. 163-166, 2012.

[18] G.Z. Yin, M.H. Li, W.P. Li, J. Cao, and X. Li, "Model of coal gas permeability prediction based on improved BP neural network", Journal of China Coal Society, vol. 38, no. 7, pp. 1179-1183, 2013.

\footnotetext{
Received: October 13, 2014

Revised: January 20, 2015

Accepted: June 23, 2015

(c) Zhu et al.; Licensee Bentham Open.

This is an open access article licensed under the terms of the Creative Commons Attribution Non-Commercial License (http://creativecommons.org/licenses/by-nc/3.0/) which permits unrestricted, non-commercial use, distribution and reproduction in any medium, provided the work is properly cited.
} 